\title{
La tensión umbral para la fluencia, en un material de base magnesio procesado por pulvimetalurgia
}

\author{
J.A. del Valle* y O.A. Ruano*
}

Resumen

Se ha estudiado la presencia de una tensión umbral, en una aleación de magnesio AZ31 de pequeño tamaño de grano conteniendo diferentes cantidades de partículas, procesada por métodos pulvimetalúrgicos. Se observó que el deslizamiento en fronteras de grano es el mecanismo que controla la deformación, en un intervalo amplio de velocidades de deformación y temperaturas. La disminución drástica de la velocidad de deformación, con una pequeña disminución de la tensión, permite deducir la presencia de una tensión umbral. Esta tensión umbral es independiente de la temperatura. En este trabajo se hace un análisis de su presencia y de su dependencia con los parámetros estructurales y mecánicos de las aleaciones.

\section{Threshold stress for creep in a magnesium based material processed by powder metallurgy}

\begin{abstract}
The presence of a threshold stress in the fine grained magnesium alloy AZ31 containing different amounts of particles and processed by powder metallurgy methods has been investigated. Grain boundary sliding has been observed to be the controlling mechanism in a wide range of strain rates and temperatures. The drastic reduction in strain rate with a small decrease in stress suggests the presence of a threshold stress. This threshold stress is independent of temperature. This paper provides an analysis of the threshold stress and its dependence on the structural and mechanical parameters.
\end{abstract}

Keywords

Creep; Threshold stress; Composite; Magnesium; Powder metallurgy.

\section{INTRODUCCION}

Cada vez, es mayor el interés de las aleaciones de magnesio como materiales estructurales. Para mejorar su resistencia es usual procesar el material para obtener un tamaño de grano fino. Con el mismo propósito, se suele hacer precipitar fases finas y estables, o procesarlas mediante métodos pulvimetalúrgicos para introducir partículas finas y estables.

Es usual observar que el mecanismo que controla la deformación en materiales de grano fino a temperaturas medias y altas es el deslizamiento de fronteras de grano, DFG. Si el proceso de acomodación de este mecanismo es el movimiento de dislocaciones, entonces, es de esperar que las partículas afecten a la deformación y que éstas puedan producir un efecto de tensión umbral, al no permitir el DFG por debajo de una cierta tensión. Sin embargo, esta tensión debería ser prácticamente independiente de la temperatura ya que la únicas contribuciones térmicas que se producen deben ser aquellas proveniente de la variación del módulo de elasticidad y de la difusividad. Por lo tanto, una tensión umbral dependiente de la temperatura y del tamaño de grano no tendría sentido físico.

En diversos trabajos que analizan la deformación a alta temperatura, de aleaciones pulvimetalúrgicas de base magnesio con grano fino ${ }^{[1-7]}$, no queda claro la dependencia con la temperatura de la tensión umbral. Esta incertidumbre, también, existe en distintos estudios con otros materiales, en especial, aleaciones de base aluminio reforzadas por partículas ${ }^{[8-11]}$. Este trabajo es una contribución para dilucidar estos aspectos, concernientes a la tensión umbral en materiales metálicos reforzados.

*Departamento de Metalurgia Física, CENIM, CSIC, Avda. de Gregorio del Amo 8, 28040 Madrid. e-mail: delvalle@cenim.csic.es; ruano@cenim.csic.es. 


\section{METODO EXPERIMENTAL}

Se obtuvieron dos materiales pulvimetalúrgicos basados en la aleación de magnesio AZ31. Primeramente, se obtuvieron polvos a partir de barras coladas de la aleación AZ31, usando la técnica de Electrode Induction-melting Gas Atomization (EIGA). El tamaño medio de partícula de polvo fue de $100 \mathrm{~mm}$. A partir del polvo atomizado se procesó el primer material pulvimetalúrgico, denominado AZ31-PM, mediante una ruta de procesado consistente en una compactación isostática en frío a $340 \mathrm{MPa}$ y una extrusión en caliente a $350^{\circ} \mathrm{C}$ con una relación de extrusión de 1:25 y una velocidad de 0,4 $\mathrm{mms}^{-1}$.

En el caso del segundo material, denominado AZ31-PMCM, el polvo de metal fue molido en un ambiente de nitrógeno líquido, utilizando un molino de tipo attritor, modelo Union Process 01HD. Las condiciones de molienda fueron relación de masa polvo/bolas 1:10, tamaño de bola 3/16", velocidad de molienda $1.000 \mathrm{rpm}$ y $1 \mathrm{~h}$ de tiempo de molienda. Posteriormente, los polvos fueron consolidados utilizando la misma ruta que en el caso del material AZ31-PM.

En este trabajo, se comparan las propiedades mecánicas a alta temperatura de ambos materiales pulvimetalúrgicos de base AZ31 con datos experimentales, previamente publicados ${ }^{[12 \text { y } 13]}$, de la aleación AZ31 colada y laminada, provista por Magnesium Elektron en la condición "O", con un tamaño de grano de $18 \mathrm{~mm}$.

La microestructura de los materiales preparados se examino mediante microscopía óptica. La preparación de las muestras consistió en desbaste mecánico con lijas de $\mathrm{SiC}$, seguido de pulido con pasta diamantada de 6 y $1 \mathrm{~mm}$ y, finalmente, un pulido final con sílice coloidal. La estructura de grano se reveló utilizando un ataque químico con una solución de etanol (100 ml), ácido pícrico (2 g), ácido acético $(2 \mathrm{ml})$ y agua $(5 \mathrm{ml})$. El tamaño medio de grano se determinó utilizando el método de intercepción lineal midiendo, como mínimo, 1.000 granos por muestra. El tamaño de grano verdadero se calculó multiplicando la longitud de intercepción media por un factor 1.74 . Se prepararon muestras para microscopia electrónica de transmisión (TEM) utilizando un electropulido con una solución de 25 $\%$ de ácido nítrico en metanol y, finalmente, un abrillantado por bombardeo de iones.

El comportamiento mecánico se estudió mediante ensayos de tracción a temperaturas de 300, 350 y 400 ${ }^{\circ} \mathrm{C}$ y velocidades de deformación en el intervalo $2 \mathrm{x}$ $10^{-5}-10^{-1} \mathrm{~s}^{-1}$. Se utilizaron muestras cilíndricas con longitud útil de $10 \mathrm{~mm}$ y radio $3 \mathrm{~mm}$, mecanizadas con eje de tracción paralelo a la dirección de extrusión. Los ensayos de tracción se llevaron a cabo en una máquina universal de ensayos mediante tornillo, equipada con un horno parabólico; el tiempo de calentamiento y estabilización de la temperatura fue de $30 \mathrm{~min}$. Se realizaron dos tipos de ensayos de tracción, a velocidad de deformación constante y ensayos con cambios en la velocidad de deformación, descritos previamente ${ }^{[12}$ y 14] . Estos ensayos de saltos se realizaron usando una velocidad constante hasta llegar al la tensión máxima y, a continuación, saltos a las diferentes velocidades de deformación estudiadas, con el objeto de determinar las tensiones de estado estacionario correspondientes sin cambios importantes en la micro/subestructura.

\section{RESULTADOS Y DISCUSION}

La tabla I muestra tamaños medio de grano de los materiales pulvimetalúrgicos, para el material obtenido después de la extrusión y medido en la zona útil de probetas después de ser ensayado a distintas temperaturas. En la figura 1 a) y b) se muestran micrografías del material preparado a partir de: polvo sin moler y molido, respectivamente. Ambos materiales presentaron tamaños de grano similares y mostraron partículas dispersas de óxido de magnesio, $\mathrm{MgO}$. La fracción en volumen de las partículas de $\mathrm{MgO}$ fue mayor en el material procesado por crío-molienda (Fig. 1 c)).

Tabla I. Tamaño medio de grano de los materiales pulvimetalúrgicos de la aleación AZ31 antes y después de los ensayos a alta temperatura

Table I. Mean grain sizes of the AZ31-based powder-metallurgy materials before and after tensile testing at high temperature

\begin{tabular}{|c|c|c|c|c|}
\hline Muestra & $\begin{array}{c}\mathrm{d}[\mathrm{\mu m}] \\
\text { Extruído }\end{array}$ & $\begin{array}{c}\mathrm{T}\left[{ }^{\circ} \mathrm{C}\right] \\
\text { Ensayos } \\
\text { de tracción }\end{array}$ & $\begin{array}{c}\mathrm{d}[\mu \mathrm{m}] \\
\text { Después } \\
\text { del ensayo }\end{array}$ & $\sigma_{0}[\mathrm{MPa}]$ \\
\hline \multirow{3}{*}{ AZ31-PM } & \multirow{3}{*}{3,6} & 300 & 3,5 & 6,5 \\
\hline & & 350 & 5 & 5,6 \\
\hline & & 400 & 11,4 & 4,5 \\
\hline \multirow{3}{*}{$\begin{array}{c}\text { AZ31-PM- } \\
\text { Molido }\end{array}$} & \multirow{3}{*}{3,7} & 300 & 3,6 & 19,8 \\
\hline & & 350 & 4,2 & 19,4 \\
\hline & & 400 & 9,6 & 19,1 \\
\hline
\end{tabular}



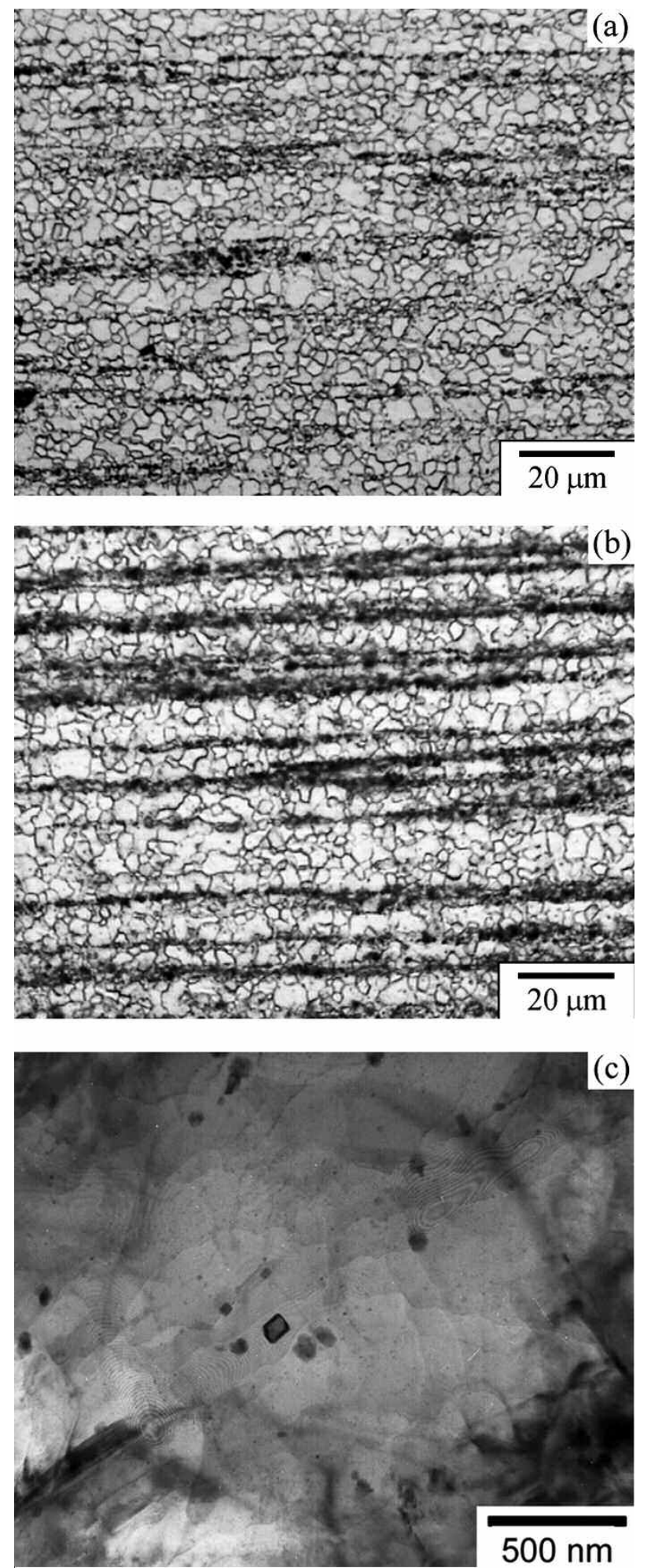

Figura 1. Micrografías ópticas de los materiales preparados a partir de polvo de la aleación AZ31 obtenidos con: (a) polvo sin moler; (b) polvo molido; (c) polvo molido, TEM.

Figure 1 Optical micrographs of materials processed from powders of the AZ31 alloys obtained from: (a) as-atomized powder; (b) milled powder; (c) milled powder, TEM.
La figura 2 a) muestra curvas velocidad de deformación frente a tensión a diferentes temperaturas y para dos materiales compuestos, uno, obtenido a partir de polvo sin moler (símbolos cuadrados) y, el otro, a través de polvo molido (símbolos redondos). Se observa claramente, en ambos materiales, una disminución drástica de la velocidad de deformación con una pequeña disminución de la tensión. Esta disminución es igual para todas las temperaturas y tiende hacia una tensión de, aproximadamente, $20 \mathrm{MPa}$ para el material molido y 5-7 MPa para el material sin moler, por lo que se suele decir que estas tensiones corresponden a una tensión umbral. En contraste, la resistencia a la fluencia de los dos materiales, en la región de velocidades de deformación comprendidas entre $3 \times 10^{-3}$ y $10^{-1} \mathrm{~s}^{-1}$, es similar. En esta región, se observa que el exponente de la tensión, n, tiene un valor bajo, cercano a 2. La figura 2 b) muestra los mismos datos para una chapa de AZ31 y sirve como comparación.

La figura 2 c) muestra cómo se determina la tensión umbral para la aleación AZ31-PM molida y sin moler, representándose frente a la tensión la velocidad de deformación elevada al inverso del exponente de la tensión correspondiente al mecanismo controlador, en este caso, $\mathrm{n}=2$. La tensión umbral se determina a partir de la intersección con el eje de tensión. Se obtienen, así, valores cercanos a 5,5 MPa para el material sin moler y, aproximadamente, 19,5 MPa para el material molido.

Con estos datos, se puede suponer que el comportamiento de los materiales se rige por una ecuación potencial típica de la superplasticidad y el deslizamiento de fronteras de grano del tipo:

$$
\dot{\varepsilon}=A D\left(\frac{b}{d}\right)^{p}\left(\frac{\sigma-\sigma_{o}}{E}\right)^{n}
$$

donde, $\dot{\varepsilon}$ es la velocidad de deformación, A es una constante del material, $\mathrm{D}$ es la difusividad apropiada (siendo $\mathrm{D}=\mathrm{D}_{0} \exp (-\mathrm{Q} / \mathrm{RT})$ y $\mathrm{D}_{0}$ una constante, $\mathrm{Q}$, la energía de activación apropiada, $\mathrm{R}$, la constante de los gases y $\mathrm{T}$, la temperatura absoluta), b es el vector de Burgers, d es el tamaño de grano, p es el exponente del tamaño de grano, ? es la tensión aplicada, $\sigma_{0}$ es una tensión umbral, E es el módulo de Young y $\mathrm{n}$ es el exponente de la tensión. Típicamente, para un mecanismo de fluencia por movimiento de dislocaciones, $\mathrm{D}$ es igual a la difusividad en volumen, $\mathrm{p}=0$ y $\mathrm{n}=5$, mientras que para un mecanismo de deslizamiento de fronteras de grano (DFG), $\mathrm{n}=2$, y $\mathrm{D}$ es igual a la difusividad en volumen o a lo largo de fronteras de grano, en cuyo caso, $\mathrm{p}=2$ o 3 , respectivamente. Salvo en casos excepcionales, como es el de 
(a)

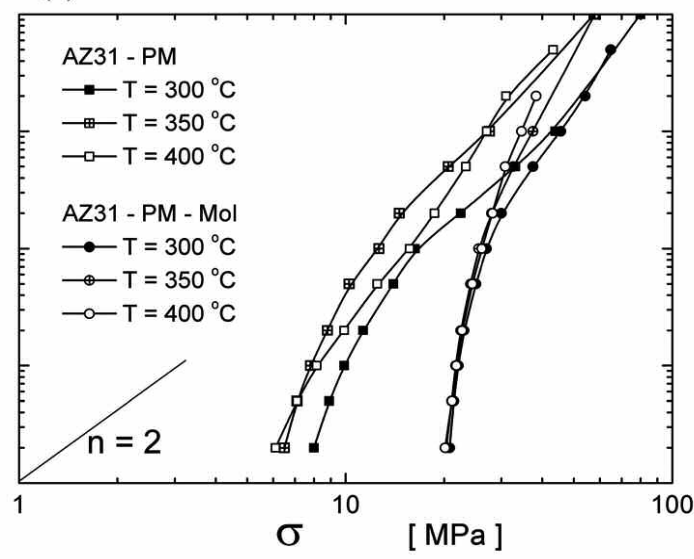

(c)

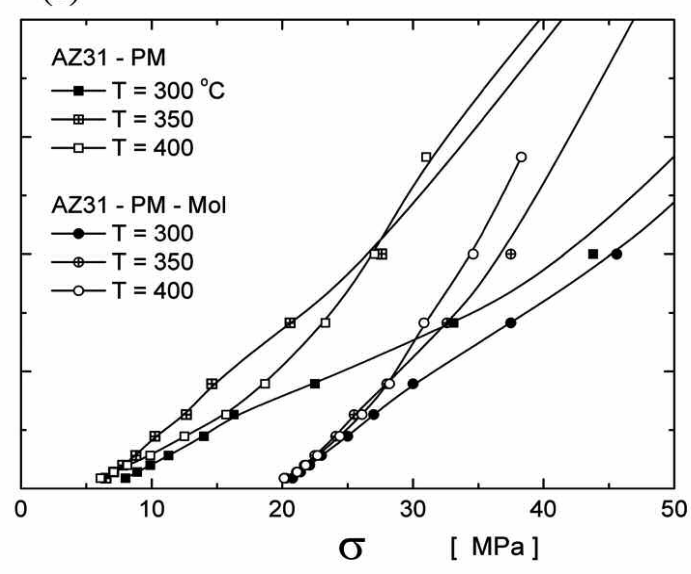

(b)

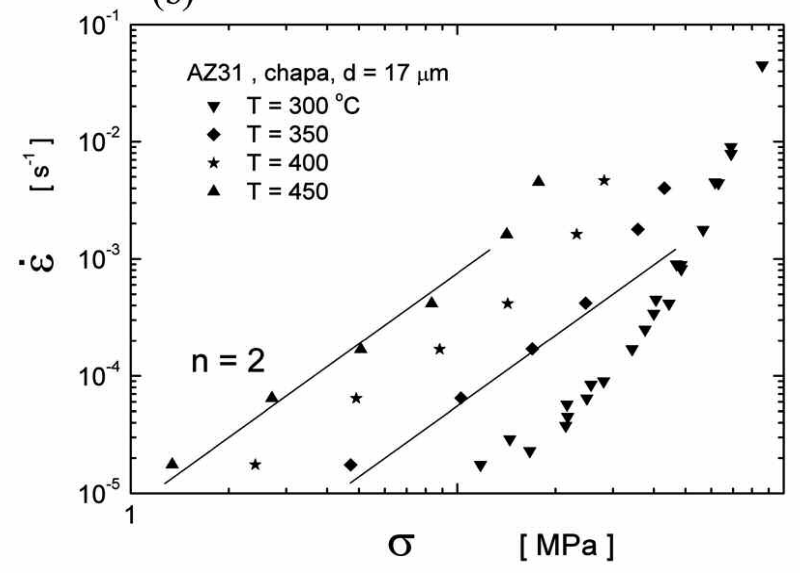

(d)

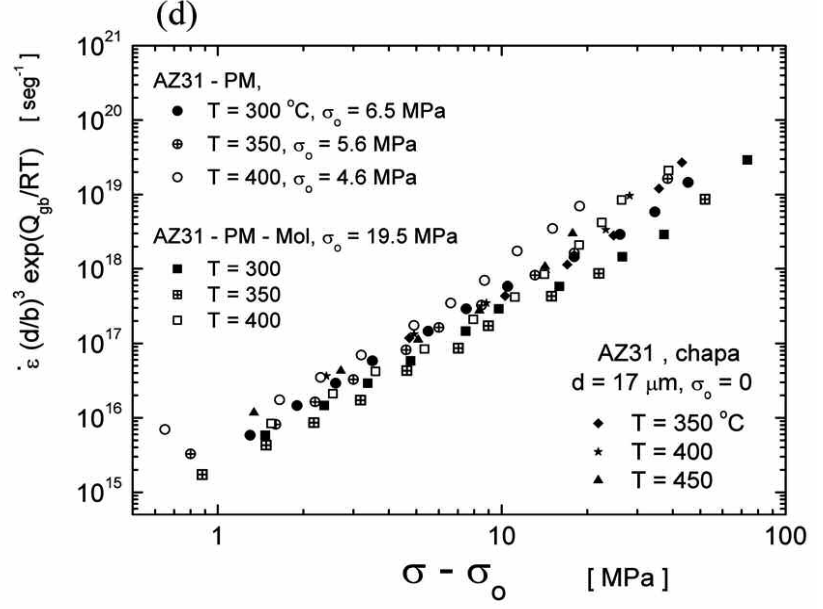

Figura 2. a) y b) Curvas velocidad de deformación en función de la tensión para los materiales PM y chapa, respectivamente; c) Determinación de la tensión umbral; d) Datos del material PM sin moler y molido, y de chapa, compensados por la difusividad a lo largo de fronteras de grano y el tamaño de grano inicial.

Figure 2. a) y b) Strain rate vs. stress curves for PM and sheet materials respectively; c) Plot for the determination of the threshold stress; $d$ ) Strain rate compensated by diffusivity along grain boundaries and grain size for PM and sheet materials.

los materiales compuestos, la tensión umbral es considerada igual a cero.

En la figura 2 d) se presentan los datos del material pulvimetalúrgico sin moler y molido, mostrados en la figura 2 a), usando una representación en donde se compensa la velocidad de deformación por la difusividad a lo largo de fronteras de grano $\left(Q_{\mathrm{gb}}=92 \mathrm{~kJ} / \mathrm{mol}\right)$ y el tamaño de grano, utilizando un valor del exponente $\mathrm{p}=3$. Para comparar, se incluyen datos a diferentes temperaturas de la chapa laminada de la aleación AZ31 con un tamaño de grano de $17 \mu \mathrm{m}$. Se puede apreciar que, en el caso de la chapa laminada, la compensación con el factor de difusividad a lo largo de fronteras de grano permite que los datos tiendan a una única curva con pendiente $\mathrm{n}=2$. En el caso de los materiales pulvimetalúrgicos se observa $\mathrm{n}=2$ a tensiones altas, convergiendo a los valores de la chapa laminada. Esto es una indicación de que el mecanismo que controla la deformación es el DFG.

Es interesante comparar estos resultados con otros provenientes de la literatura. La figura 3 presenta valores de la tensión umbral en función de la temperatura, para diversas investigaciones realizadas en materiales de base magnesio. Al igual que los resultados obtenidos por los autores del presente estudio, algunos trabajos muestran una tensión umbral prácticamente independiente de la temperatura. Esta tensión umbral tiene un sentido físico en el caso del deslizamiento de fronteras de grano. Consistiría en asumir que la presencia de los reforzantes supone un impedimento a este deslizamiento, aumentando este efecto al aumentar su concentración. 


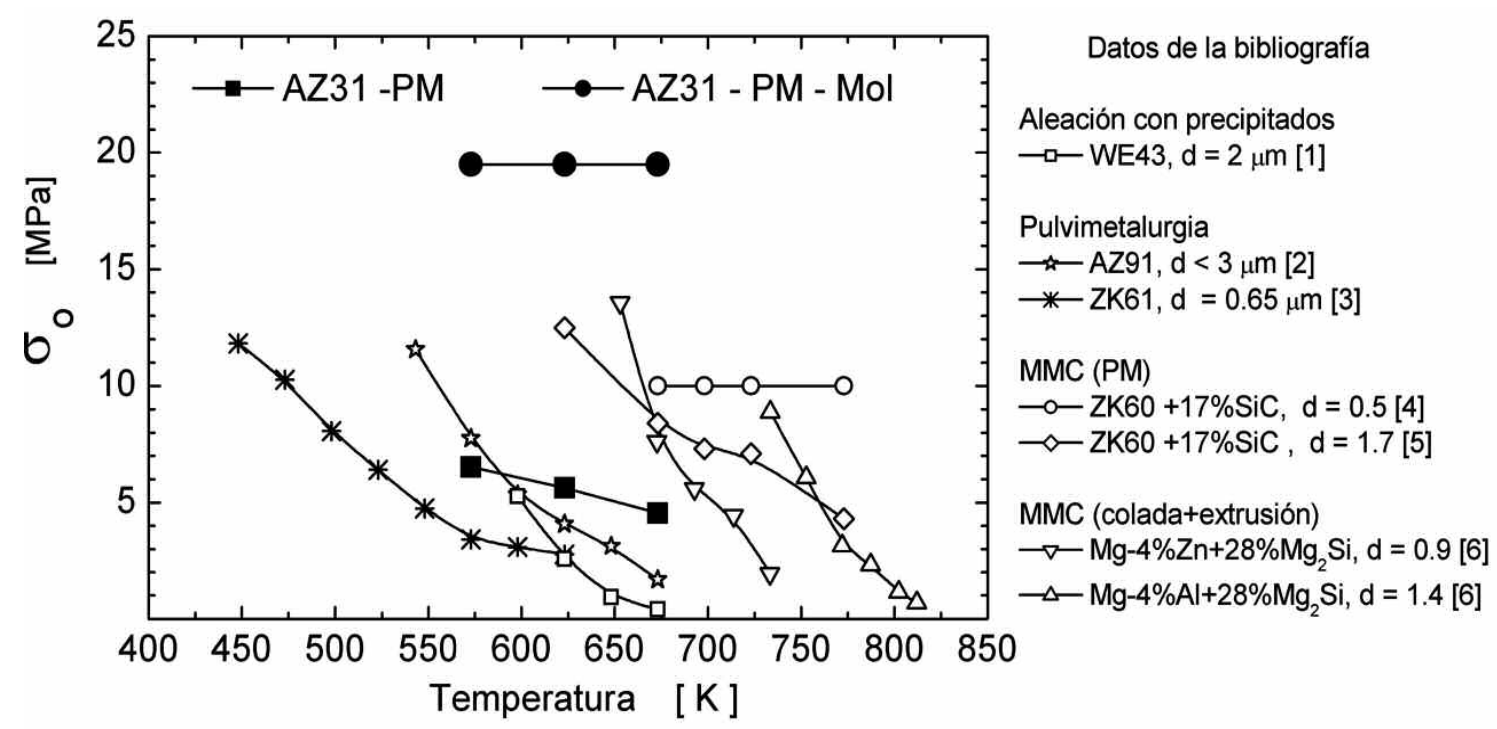

Figura 3. La tensión umbral en función de la temperatura para diversos trabajos de la literatura.

Figure 3. Threshold stress as a function of temperature for several investigations of the literature.

Sin embargo, la dependencia con la temperatura de la tensión umbral, que se observa en algunos trabajos representados en la figura 3, debe llevar a una reflexión ya que, asimismo, suele existir, también, una dependencia de esta tensión umbral con el tamaño de grano. La introducción de una tensión umbral independiente de la temperatura en la ecuación (1), como se mencionó anteriormente, no supone merma alguna del sentido físico del mecanismo de deformación que, en este caso, es el de deslizamiento de fronteras de grano. Por el contrario, introducir una tensión umbral dependiente de la temperatura y del tamaño de grano hace que la ecuación (1) carezca de sentido físico ya que la dependencia con la temperatura, de la velocidad de deformación, viene dada por la difusividad, D, y con el tamaño de grano, a través del exponente del tamaño de grano, p. Existiría, por tanto, una doble dependencia con estos parámetros. También, es engañoso el término porque no existe una "tensión umbral", sino múltiples "tensiones umbrales", una para cada temperatura y tamaño de grano. Además, los diferentes autores utilizan estas tensiones umbrales según su conveniencia para ajustar las curvas y no existe un patrón que permita darles un sentido físico.

Por otro lado, un análisis de los resultados de los trabajos mencionados en la figura 3 permite sacar consecuencias importantes sobre las tensiones umbrales obtenidas. Estas tensiones se suelen determinar mediante la extrapolación a $\varepsilon=0$, en una representación $(\dot{\varepsilon})^{1 / n}$ frente a $\sigma$, similar a la mostrada en la figura 2 c). Para obtener estos valores es necesario asumir un valor concreto de $\mathrm{n}$ que, en el caso del DFG, es igual a 2. La elección de un valor determinado de $n$ es problemática porque, usualmente, hay pocos puntos experimentales y esto puede afectar, fuertemente, el valor calculado de la tensión umbral. Además de ser arbitrario el valor de n escogido, muchos materiales muestran un cambio en el valor de $\mathrm{n}$, debido, a menudo, a la interacción de otro mecanismo de deformación, frecuentemente, movimiento de dislocaciones. Otro efecto que puede llevar a la suposición errónea de una tensión umbral dependiente de la temperatura y del tamaño de grano es el crecimiento de grano que se produce durante la deformación, especialmente en la región de velocidades de deformación bajas donde las probetas están largo tiempo sometidas a deformación y a temperatura. Este crecimiento de grano afecta fuertemente a la forma de las curvas tensión-deformación debido a la fuerte dependencia con el tamaño de grano, en el mecanismo de DFG.

Específicamente, los datos de la referencia ${ }^{[4]}$ no muestran una tensión umbral dependiente de la temperatura. Tampoco, la muestra los datos de la referencia $^{[5]}$, excepto a altas temperaturas, donde los cambios microestructurales pueden afectarla. Por otro lado, los valores de la referencia ${ }^{[1]}$ corresponden directamente a los dados por los autores, apreciándose valores bajos de la tensión umbral para todas las temperaturas aunque la velocidad de deformación del material reforzado es 50 veces menor que la del material sin refuerzo; hay que hacer notar que los valores de las tensiones umbrales tienen una fuerte dispersión, según se observa en la figura 6 de 
la referencia ${ }^{[5]}$. Respecto a la referencia ${ }^{[3]}$., los datos muestran una tensión umbral independiente de la temperatura a las cuatro temperaturas más altas, mientras que es dependiente de la temperatura a las cuatro temperaturas más bajas. Respecto a la referencia $^{[2]}$, la extrapolación de los datos dan valores de la tensión umbral dependientes de la temperatura; habría, también, que explicar la dependencia similar en el material sin refuerzo; en ambos casos, la extrapolación se realiza desde valores de velocidades de deformación, en donde $\mathrm{n}$ es, ligeramente, superior a $5 \mathrm{y}$, por lo tanto, no existe un indicio claro de una tensión umbral, lo que puede inducir a un error considerable en su determinación. Por último, en la referencia ${ }^{[6]}$ se hacen ensayos a altas velocidades de deformación y la extrapolación se hace desde un valor mínimo de $10^{-3} \mathrm{~s}^{-1}$, el cual es muy alto para dar valores fiables de la tensión umbral.

Por último, la figura 4 muestra datos de velocidad de deformación y tensión para materiales PM, PMCM y chapa de AZ31, comparados con materiales PM superplásticos y aleaciones de colada resistentes a la fluencia ${ }^{[3,5,15}$ y 16]. Se observa que las aleaciones pulvimetalúrgicas investigadas en este trabajo, para una tensión constante, fluyen a velocidades de deformación menores en el intervalo superplástico que otras aleaciones pulvimetalúrgicas de tamaño de grano similar. Por otro lado, el comportamiento de la aleación PMCM se aproximaría al de las aleaciones resistentes a la fluencia, a tensiones por debajo de, aproximadamente $20 \mathrm{MPa}$.

\section{CONCLUSIONES}

Se ha analizado el comportamiento a alta temperatura de una aleación de magnesio AZ31 de pequeño tamaño de grano conteniendo diferentes cantidades de partículas, que fue procesada por métodos pulvimetalúrgicos. La deformación del material está controlada por el deslizamiento de fronteras de grano y su comportamiento permite asumir una tensión umbral independiente de la temperatura. Una comparación con otros trabajos de la literatura sobre aleaciones de magnesio reforzadas, permite poner en duda la existencia de una tensión umbral dependiente tanto de la temperatura como del tamaño de grano. El procesado de la aleación AZ31 mediante una ruta pulvimetalúrgica con molienda mecánica se puede utilizar para transformar esta aleación en un material resistente a la fluencia, para tensiones aplicadas menores que $20 \mathrm{MPa}$.

\section{Agradecimientos}

Se agradece a la CICYT por la financiación de este trabajo a través del Proyecto MAT2009-14452.

\section{REFERENCIAS}

[1] H. Watanabe, T. Mukai, K. Ishikawa, T. Mohri, M. Mabuchi y K. Higashi, Mater. Trans. 42 (2001) 157-162.
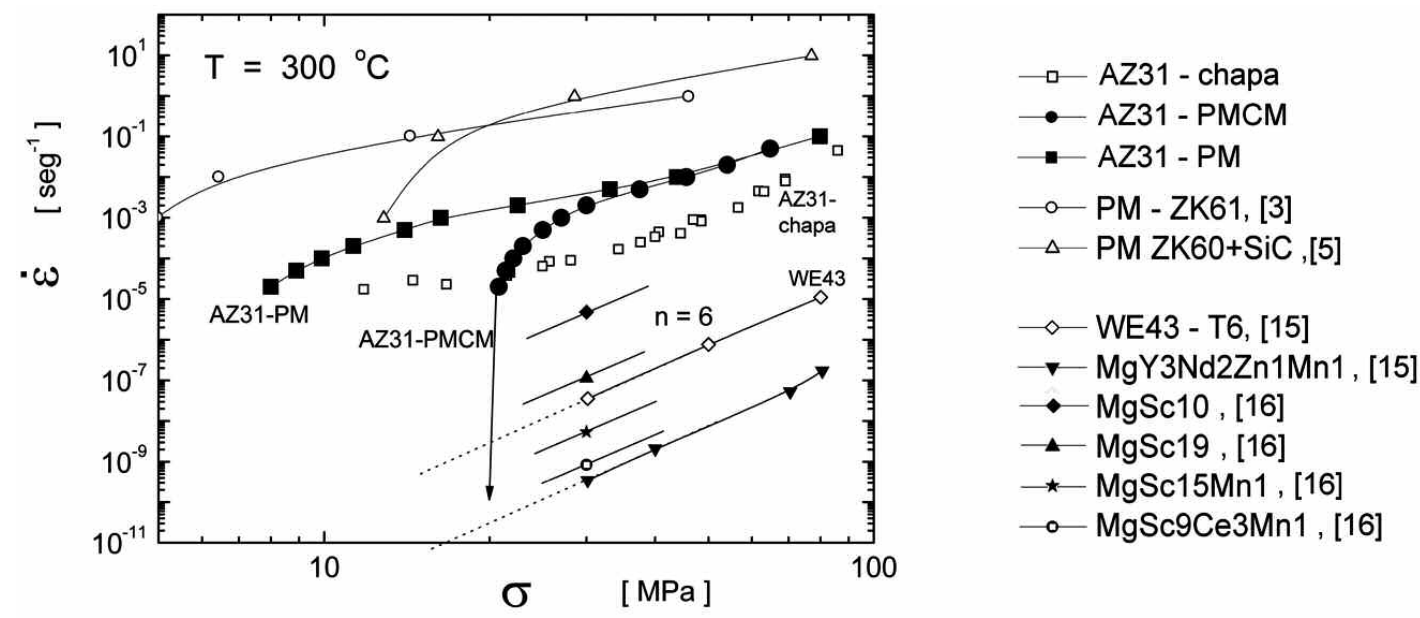

Figura 4. Curvas velocidad de deformación en función de la tensión para los materiales PM y chapa de AZ31 estudiados en este trabajo, comparados con materiales PM superplásticos y aleaciones de colada resistentes a la fluencia.

Figure 4: Strain rate vs. stress curves for the AZ31 PM and sheet materials studied in this work, compared against superplastic PM materials and creep resistant cast alloys. 
[2] M. Mabuchi, T. Asahina, H. Iwasaki y K. Higashi, Mater. Sci. Technol. 13 (1997) 825831.

[3] H. Watanabe, T. Mukai, K. Ishikawa, M. Mabuchi y K. Higashi, Mater. Sci. Eng. A 307 (2001) 119-128.

[4] T.G. Nieh, A.J. Schwartz y J. Wadsworth, Mater. Sci. Eng. A 208 (1996) 30.

[5] T. Mukai, T.G. Nieh, H. Iwasaki y K. Higashi, Mater. Sci. Technol. 14 (1998) 32-35.

[6] M. Mabuchi y K. Higashi, Philos. Mag. A 74 (1996) 887-905.

[7] J.A. del Valle, F. Carreño y O.A Ruano, Scripta Mater. 57 (2007) 829-832.

[8] R. S. Mishra, T.R. Bieler y A.K. Mukherjee, Acta Mater. 45 (1997) 561-568.
[9] W. J. Kim, E. Taleff y O.D. Sherby, Scripta Metall. Mater. 32 (1995) 1.625-1.630.

[10] Y. Li y T. G. Langdon, Acta Mater. 47 (1999) 3.395-3.403.

[11] N. Du, A.F. Bower, P.E. Krajewski y E M. Taleff, Mater. Sci. Eng. A 494 (2008) 86-91.

[12] J.A. del Valle, M.T. Pérez-Prado y O.A. Ruano, Metall. Mater. Trans. A 36 (2005) 1.427-1.438.

[13] J.A. del Valle, F. Peñalba y O.A. Ruano, Mater. Sci. Eng. A 467 (2007) 165-171.

[14] J.A. del Valle y O.A. Ruano, Acta Mater. 55 (2007) 455-466.

[15] F. Hnilica, V. Janik, B. Smola, I. Stulíková y V. Očenášek, Mater. Sci. Eng. A 489 (2008) 93-98.

[16] B.L. Mordike, I Stulítková y B. Smola, Metall. Mater.Trans. A 36 (2005) 1.729-1.736. 